\title{
Functional and Behavioral Adaptation After Intermittent Hypobaric Hypoxia Induction in Sprague-Dawley Rats Brain
}

\author{
Fanny S. Farhan \\ Departement of physiology \\ Faculty of Medicine and Health \\ University of Muhammadiyah \\ Jakarta, Indonesia
}

Wawan Mulyawan

Indonesian Air Force Institute of Aviation Medicine

Jakarta, Indonesia

\author{
Mohamad Sadikin, Sri WA. Jusman \\ Centre of Hypoxia and Oxidative Stress Study \\ Departement of Biochemistry and Biology Molecular \\ Faculty of Medicine, University of Indonesia \\ Jakarta, Indonesia
}

\begin{abstract}
Intermittent hypobaric hypoxia (IHH) is believed to have neuroprotective effect. IHH induces changes in gene expression and intracellular signaling pathways that lead to the emergence of intracellular adaptation through HIF-1 alpha gene activity. IHH induction decreased brain cortical tissue damage, and increased microvascular density. Further study is needed to understand physiological and behavioral response after IHH induction. The aim of this study is to investigate the effect of IHH on neuronal function in conducting neurotransmitters. A total of 25 Sprague-Dawley rats were divided into 5 groups: 1 group as control and 4 groups exposed to hypobaric hypoxia (HH). 1 group were exposed to single $\mathrm{HH}$, and 3 groups exposed to $\mathrm{HH}$ intermittently (1 week interval) in Indonesian Air Force Institute of Aviation Medicine using hypobaric chamber. After the induction, the rat brain was taken for immunochemistry analysis. The level of GABAA receptor and glutamate receptor (NMDAR) decreased significantly in single $\mathrm{HH}$ group, but raised significantly after intermittent induction compare to control group $(\mathbf{p}<0.05)$. IHH induction shows adaptation respons in neurotransmitter conduction in Sprague-Dawley rats brain.
\end{abstract}

Keywords-Attitude; Contraceptions; Family Planning; Knowledge; IUD

\section{INTRODUCTION}

Hypoxia at early stage will caused responses of the cardiovascular and respiratory system, and if the condition continued would lead pathological effect to target organ such as the brain. Hypoxia is not always cause damages. Sublethal exposure to hypoxia known as hypoxia preconditioning is believed to have neuroprotective effect. Hypoxia preconditioning induces changes in gene expression and intracellular signaling pathways that lead to the emergence of intracellular adaptation through the process of erythropoiesis, angiogenesis, glucose transport and anaerobic glycolysis through HIF- 1 alpha gene activity $[1,2]$.
Intermittent hypobaric hypoxia (IHH) is part of hypoxia preconditioning by exposing to sublethal hypoxia on barometric low pressure and repeated within a certain period. The literature describes that induction to $\mathrm{IHH}$ also provide neuroprotective effect. IHH induction decreased brain cortical tissue damage and increased microvascular density $[3,4]$.

IHH trigger presynaptic neuron release glutamate resulting in depolarization, increased $\mathrm{Ca}^{2+}$ in the post-synaptic neuron. Increased $\mathrm{Ca}^{2+}$ induces many molecules that play an important role in the process of synaptic plasticity activate intracellular signaling cascade that occurs neuroplasticity and ultimately lead to the growth of new dendrites The emergence of new dendrites bulge is thought to increase and improve the structure and function of neurons due to unfavorable conditions occurring on the network such as hypoxia, brain injury and others [5-7].

The aim of this study is to investigate neuronal adaptation after induction of IHH measuring the expression of glutamate receptors (NMDA) and GABA receptors as one of the major neurotransmitter in the brain tissue.

\section{METHODS}

\section{A. Design Experiment}

This experimental animal study was conducted in the Indonesian Air Force Institute of Aviation Medicine (Lakespra Saryanto). A total of 25 Sprague Dawley rats were divided into 5 different groups. Control group without exposure to hypobaric hypoxia, group 1 exposed to single exposure hypobaric hypoxia (HH/day-1), group 2 exposed to 2 period of hypobaric hypoxia (IHH 1 time intermittently/day-1 and 8), group 3 exposed to 3 times hypobaric hypoxia (IHH 2 times intermittently/day $1,8,15)$, group 4 exposed to 4 period of hypobaric hypoxia (IHH 3 times intermittently/day 1,8, 15, 22). After acclimatization to room temperature of $25^{\circ} \mathrm{C}$ for a week, group 1-4 were exposed to type I hypobaric chamber training 
until $35,000 \mathrm{ft}$ of altitude, then descending gradually to 30,000 $\mathrm{ft}, 25,000 \mathrm{ft}$ and 18,000 ft of altitude (Fig.1). After the induction, the brain was taken for immunochemistry analysis.

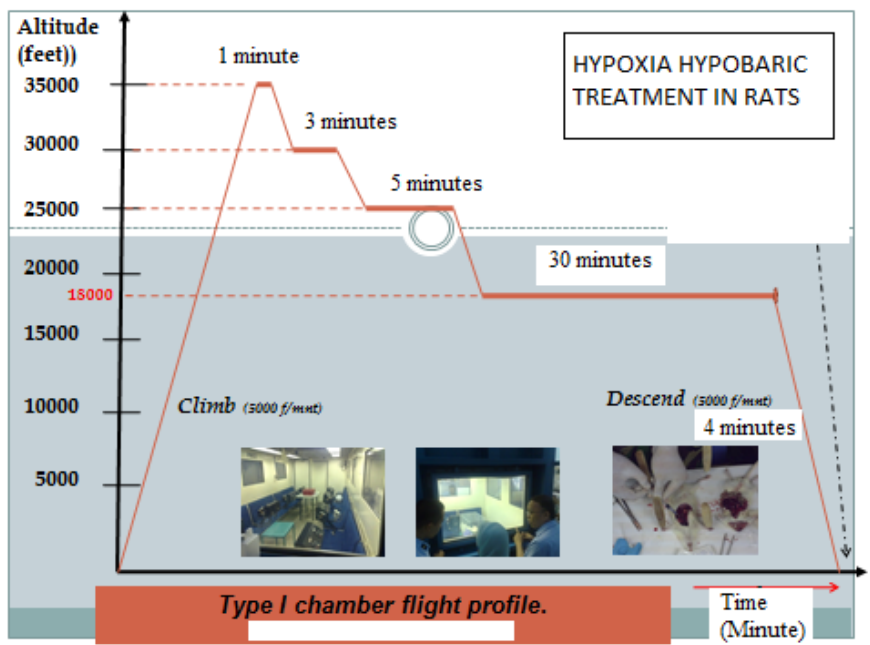

Fig. 1. The flight plan of intermittent hypobaric hypoxia exposure [8].

\section{B. Immunochemistry}

The rats were sacrificed by dislocated their heads by stretching the cervical bones from the thoracic vertebrae. The head was decapitated, the brain removed. Brain tissue was immersed in to $10 \%$ formaldehyde solution. Tissue was dehydrated in serial step of alcohol $(80 \%, 90 \%, 100 \%)$. Tissue was immersed in xylene for 2 times and immersed in liquid paraffin for 3 times, last was embedded in paraffin block. Frontal sections were cut by $5 \mu \mathrm{m}$ microtome from 1.5 to 2.5 $\mathrm{mm}$ in front of bregma. The sections were embedded on polysine coated premium. The antibody used was mouse monoclonal primary antibody glutamate receptor, N-Methyl DAspartate 2a (GRIN2A) and Anti-GABA A receptor alpha 1 antibody [S95-35]. Sections were pictured with microscope Olympus inverted IX73. Number of NMDAR and GABA receptor expression were count by optilab software.

\section{RESULTS}

A. Immunohistochemistry for glutamate receptor (NMDAR) NMDAR expression was calculated on the frontal cortex.
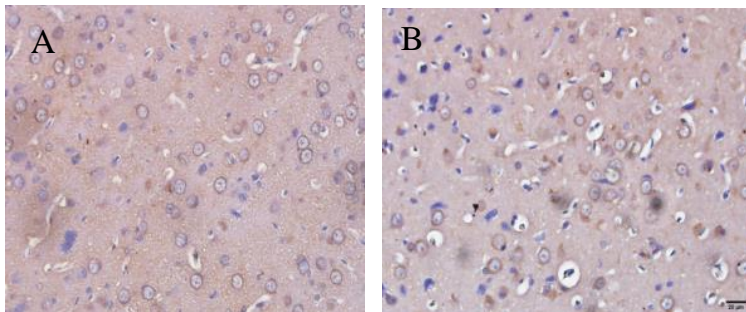
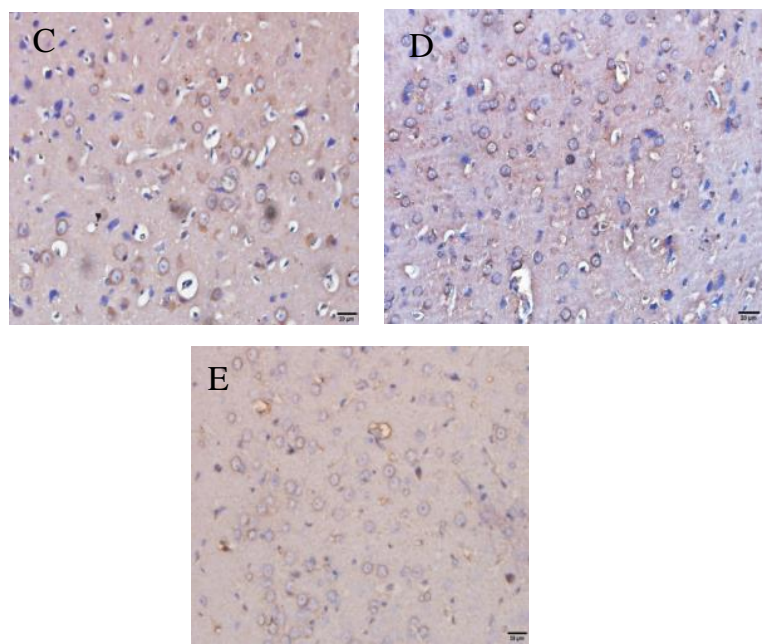

Fig. 3. IHC slide of frontal cortex (40x magnification).labelled with antibody anti NMDAR (GRIN2A) A. Control B. single hypobaric hypoxia C. hypobaric hypoxia 1 time intermittent $\mathrm{D}$. hypobaric hypoxia 2 times intermittent $\mathrm{E}$. hypobaric hypoxia 3 times intermittent

Before ANOVA test, data is tested for normality and homogeneity. One-way ANOVA results showed $p<0.05$. It can be concluded that there is a significant difference to the number of NMDA receptors after IHH induction. Fig. 3 shows that the highest number of NMDAR expression is in IHH 2 times intermittent, and the lowest is in single hypobaric hypoxia group (group 1). Post Hoc test results are significant differences between the control group with hypoxic hypobaric group, and there are significant differences between hypobaric hypoxia group with 2 and 3 times intermittent.

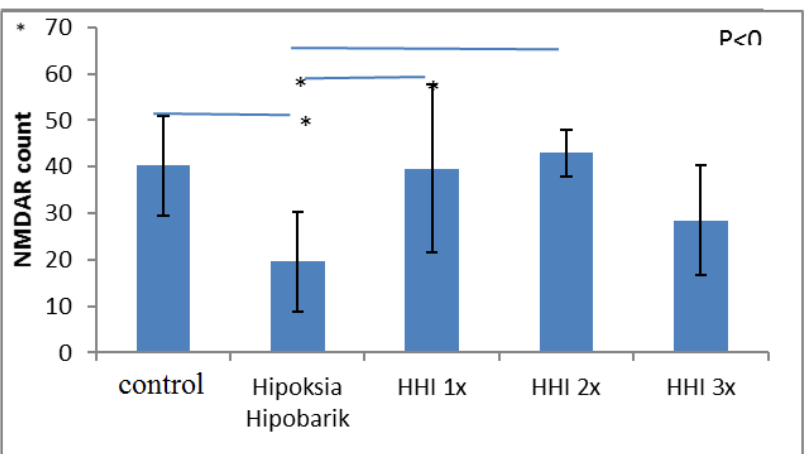

Fig. 3. ANOVA analysis for NMDAR 


\section{B. Immunohistochemistry for $G A B A_{A}$ receptor (GABRAI)}

GABRA1 expression was calculated on the frontal cortex
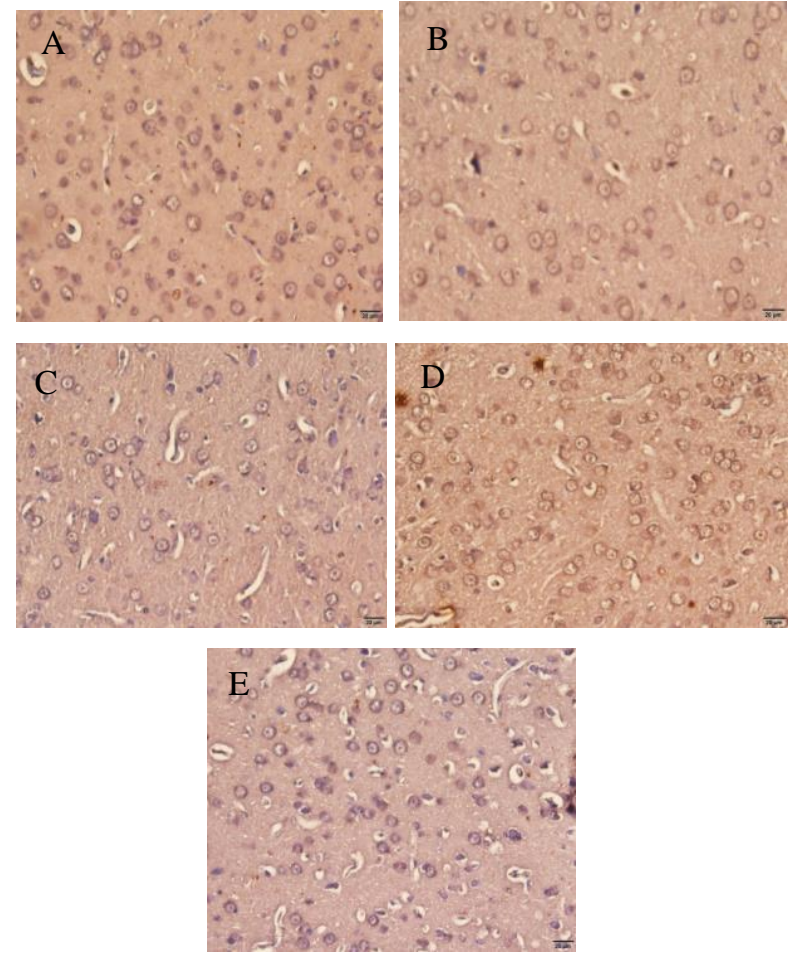

Fig. 4. IHC slide of frontal cortex (40x magnification).labelled with antibody anti GABA receptor A.Control B. hypobaric hypoxia C. single hypobaric hypoxia 1 time intermittent D. hypobaric hypoxia 2 times intermittent $\mathrm{E}$. hypobaric hypoxia 3 times intermittent

Before the ANOVA test, data is tested for normality and homogeneity. One-way ANOVA results showed $p<0.05$, it can be concluded there is a significant difference to the number of $\mathrm{GABA}_{\mathrm{A}}$ receptor cells after induction of IHH. Fig. 5 shows that the highest number of GABRA1 expression found in IHH 2 times intermittent and the lowest in single hypobaric hypoxia group. Post Hoc result significant difference between the control group with the single hypobaric hypoxia and 1 time intermittent, there are significant differences between single hypobaric hypoxia group with groups 2 and 3 times intermittent.

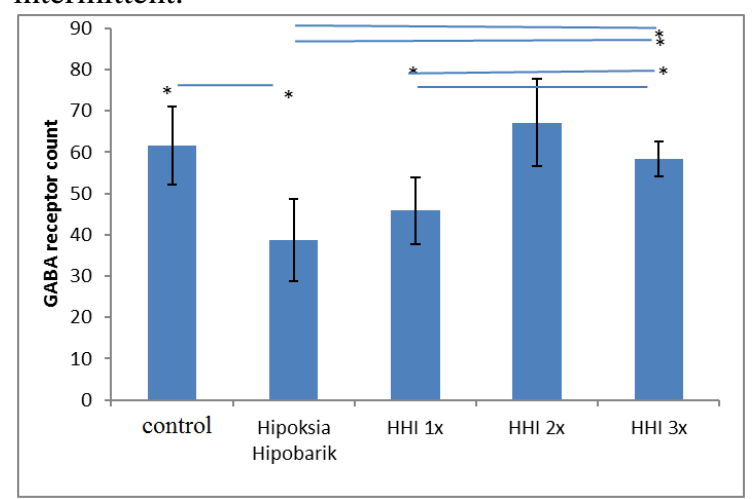

Fig. 5. ANOVA analysis for GABA receptor.

\section{DISCUSSION}

This study analyzed the expression of glutamate receptors NMDA (N-methyl-D-Aspartate) and GABA receptors (GABRA) from immunohistochemistry preparations (IHC) of brain tissue. For IHC results to be well secured, positive and negative controls need to be made. Positive control is a tissue to express antigens and negative control is a tissue that necessarily does not express antigen. This study used mice brain tissue as a positive control and brain tissue of experimental animals that were not labeled antibodies as negative controls.

Comparison between IHC tissue labeled with antibodies with negative control originated from the same tissue, showing differences in the color intensity. It was indicates that the IHC process has successfully identified the NMDA protein. IHC detects protein antigens in the tissues by using the principle of binding of specific antibodies against the antigen

NMDA and GABRA expression analysis was performed by counting the number of neuron cells that had brown membrane walls. NMDA and GABRA receptors can be seen in neuronal cells, glial and throughout the brain region.

When compared with the control group, NMDA expression in hypoxic hypobaric exposure decreased significantly. This raises the notion that neurotoxicity is the cause of cells death because cells have not been trained in hypoxia. Neurotoxicity caused an influx of calcium ions in large quantities, which causes the activation of nitric oxide synthase to form nitric oxide, calcium sensitive to proteases and mitochondrial damage. NO which is part of ROS caused nitration of proteins, protein oxidation, lipid peroxidation and DNA damage that ends with cells death. This fact had also been reported by Mulyawan ${ }^{8}$, stated that first exposure of hypoxia caused the degree of brain tissue damage of the most high compared with exposure to hypoxia in the next group. Brain tissue damage decreased significantly to HHI treatment in the fourth week. It was observed also by Gascon S et al, which examined the effects of cerebral ischemia excitotoxicity and against NMDA NR2B subunit and PSD NR2a and 95. The study concluded that in the conditions of ischemia there were the neuronal cell morphological changes in the form of condensation nucleus and cell body size changes. The changes that occur indicate a progressive ischemic state towards cell death.

NMDA expression increased at first treatment of HHI and reached its peak at second treatment of HHI. At third HHI exposure, NMDA expression decreased again, although the decrease was statistically not significantly different from the control group. The decrease is due to the occurrence of cellular adaptation processes to the hypoxic conditions that occur where HIF $1 \alpha$ as master swich gene has activated its downstream gene to maintain cellular oxygen availability. The pattern of NMDA receptor expression change is in line with the changing pattern of HIF $1 \alpha$ expression of HHI post exposure investigated by Mulyawan [8]. The study concluded that there was an increase of HIF $1 \alpha$ expression in hypobaric hypoxic treatment, and the expression decreased with intermittent exposure at subsequent 
HHI treatment. Although it can not be proven that NMDA expression works under the control of HIF $1 \alpha$, it can be presumed that intermittent treatment provides a protective effect of cell death due to increased oxygen and glucose delivery into neuron cells. At the last exposure, cell function has been recovered including functions related to glutamate so that the condition returns to its initial state.

Another factor that causes a decrease in neurotoxicity is in the intermittent conditions occurs a glial activation of the transporter-1 glutamate (GLT-1). This is proven by research of Gong et al [9], which concluded that exposure to HHI increases regulatory activity (GLT-1). With the activity of GLT-1, large amounts of glutamate in the extracellular can be carried by GLT-1 into astrocytes. This keeps the condition of neurotoxicity at a lower level when compared with hypoxic exposure without intermittent.

\section{A. Interaction between NMDA and GABA}

It is known that under hypoxic conditions, excitatory neurotransmitters (glutamate) are released massively from presynaptic neurons that contribute to cell death. When hypoxia is a depolarized neuron followed by an increase in extracellular potassium concentration and decrease intracellular sodium. This condition causes the synapse release glutamate neurotransmitter and activate its receptor NMDA, causing calcium into the cell, activating $\mathrm{Ca}^{2+}$ dependent enzymes, NO synthetase. Simultaneously, the release of inhibitory neurotransmitters also increased in order to compensate the increased number of excitatory/glutamic neurotransmitters. GABA's inability to cope with increased glutamate will lead to a pathological condition of seizures and may end in cell death [9].

In the correlation test between NMDA receptors and GABA, it can be seen that the correlation is positive which the increase in glutamate will be followed by an increase in GABA receptors. In the hypoxic hypobaric day treatment until the second HHI group, there was an increase in the number of cells expressing NMDA receptors, the same thing also occur in GABA receptors. In the third HHI treatment, NMDA decrease also followed by a decrease in GABA receptors. This fact shows that intermittent hypoxia provides an opportunity for nerve cells to achieve a balance between excitatory and inhibitory functions [10].

\section{B. Hypobaric Hypoxic and Intermittent Hypobaric Hypoxia}

In the hypobaric hypoxic group, oxidative stress occurs most severely. Production of ROS by mitochondria increases in the event of resistance to electron transport, then a cell membrane polarization changes that may cause a response to the Fas signal and excessive expression of Bax so that apoptosis may occur [11]. Massive calcium enhancement is also a trigger factor for ROS formation, protease enhancement, nuclease and NO. In this group, the state of severe hypoxia experienced leads to cell death due to the condition of the neurotoxicity that occurs. The rate of cortical damage in this group is the heaviest with the lowest microvascular density [12]. With severe cellular damage, it certainly interferes with the work of the NMDA and GABA receptors located on the cell membrane, so it appears that in this group the receptor expression is lowest compared to the other groups. With impaired neurotransmitter conduction, it will affect the ability of motor and cognitive function. The cognitive and motor abilities of hypobaric hypoxic groups are the worst compared to other groups. But on the other hand, hypobaric hypoxic exposure induces HIF stability 1, activating downstream genes in an effort to cope with providing sufficient energy.

If hypoxia occurs intermittently, the formation of ROS will follow intermittent pattern and cell adaptation will occur, so the reaction of using the antioxidants becomes more efficient. By decreased oxidative stress and apoptosis, as well as the stability of HIF $1 \alpha$ in the intermittent group [8], indicates that there was an adaptation to hypoxic exposure. The oxygen delivery to the cells is better, the angiogenesis process occurs which leads to improved microvascular density and decreases cellular damage. So it can be said that cellular conditions no longer lead to cell death but are already neuroprotective. The expression of NMDA and GABA receptors also undergoes adaptation due to neuroplasticity response. Under ordinary circumstances, it is very difficult to perform HHI exposure as done in hypobaric chamber. However, intermittent hypoxia conditions can be achieved by exerting an excess burden on the body for a brief period. Measurably this can be done with regular physical activity such as exercise. In this state there is relative hypoxia so that body cells are exposed to oxygen depletion conditions and are trained to deal with it. It can be expected that regular physical activity in the form of exercise will provide observable benefits to HHI exposure as we report to the brain.

\section{CONCLUSION}

Intermittent hypobaric hypoxic responds physiologically to the expression of glutamate and GABA neurotransmitters in in Sprague-Dawley rats brain.

\section{REFERENCES}

[1] G.L. Semenza," Hypoxia-inducible factor-1: Oxygen homeostasis and disease pathophysiology," Trends. Mol. Med; 2001.78: vol. 8: pp345-350

[2] U. Dirnagl, K. Becker," A Meisel. Preconditioning and tolerance against cerebral ischaemia: from experimental strategies to clinical use," Lancet Neurol; (2009). Vol.8: pp398-412

[3] D.C. Costa at al," .Intermittent Hypobaric Hypoxia Induces Neuroprotection in Kainate-Induced Oxidative Stress in Rats," Mol Neurosci (2013) 50: pp402-410

[4] Bernaudin et all," Normobaric hypoxia induced tolerance to focal permanent cerebral ischemia in association with an increased expression of hypoxia inducible factor 1 and its target genes, eritropoietin and VEGF, in the adult mouse brain," Journal of cerebral blood flow and metabolism. 2002;22:393403

[5] M. Mayadevi, G. Archana, R. Prabhu, R.V. Omkumar,"Molecular Mechanisms in Synaptic Plasticity," Roczniki Akademii Medycznej w Białymstoku (2004) Vol. 49

[6] L. Sherwood," human physiology," 2000. EGC

[7] R.S. Sundaram, , L. Gowtham, B.S. Nayak," The role of excitarory neurotransmitter glutamate in brain physiology and pathology," Asian journal of pharmaceutical and clinical research. 2012:1-7

[8] W. Mulyawan," Analysis of respond adaptation of braint tissue after intermitten hyperbaric hypoxia on: Specific analysis on expresion of hypoxia inducible factor-1 $\alpha$,". PDIB FKUI, Jakarta 2011. (Disertasi)

[9] K. Zito, V. Scheuss," NMDA Receptor Function and Physiological Modulation," Elsevier . 2009;1157-1165. 
[10] L. Hertz," The glutamate-glutamine (GABA) cycle: importance of late postnatal development and potential reciprocal interactions between biosynthesis and degradation," Front endoc. 2013;4(59): 1-16.

[11] M. Gamdzyk, et al,". Hypobaric hypoxia postconditioning reduce brain damage and improves antioxidative defense in the model of birth asphysia in 7 day old rats, "N Neurochem res. 2014;39:68-75
[12] M.G. Vorobiev," A. Rybnikova, M. Samoilov," Changes in the severity of hypoxic brain damage in rats due to hypoxic postconditioning," Neurosci behav physiol. 2013;43(1):6-9. 